\title{
Lightning severity classification utilizing the meteorological parameters: a neural network approach
}

\begin{abstract}
This paper presents a technique of predicting lightning severity on daily basis by using meteorological data. The data used is supplied by Global Lightning Network (GLN) from WSI Corporation. The input of the system consists of seven meteorology parameters which had been provided by Malaysia Meteorology Service with minimal fees. Input parameters are the Minimum Humidity, Maximum Humidity, Minimum Temperature, Maximum Temperature, Rainfall, Week and Month. The output of the system determines the severity of lightning predictions in three stages; Class1: Hazardous; Class2: Warning; and Class3: Low Risk. Two training algorithms that have been tested in this study namely the Gradient Descent with Momentum Backpropagation (traingdm) and the Scaled Conjugated Gradient Backpropagation (trainscg). The traingdm has indicated better accuracy of $70 \%$ compared to the trainscg whilst in contrast; trainscg has demonstrated approximately 4 times faster training compare to traingdm.
\end{abstract}

Keyword: Artificial neural network; Backpropagation; Scaled conjugated gradient; Lightning severity prediction 